\title{
Nemaline Myopathy: A Case Report
}

\author{
Adnan A. Mubaraki \\ Department of Medicine, Taif University, College of Medicine, Taif, Saudi Arabia
}

\section{Keywords}

Congenital myopathy $\cdot$ Nemaline rods $\cdot$ Nemaline $\cdot$ Nebulin $\cdot(N E B)$ gene $\cdot$ Weakness

\begin{abstract}
Generalized weakness in the pediatric and adolescent population is caused by many disorders that affect the neuromuscular axis. As next-generation sequencing (NGS) is becoming of high yield in replacing more invasive procedures, that is, muscle and nerve biopsy, more previously undiagnosed diseases of the muscles are now labeled with specific pathogenicity. A 16-yearold-girl diagnosed with nemaline myopathy but previously was misdiagnosed with congenital myasthenia and put-on unnecessary medications. Clinicians should be aware of congenital diseases that affect the muscles and know the importance of the NGS in reaching the correct diagnosis more so when there is a history of consanguinity.
\end{abstract}

\section{Introduction}

Skeletal muscle weakness and hypotonia are the main symptoms of congenital myopathies. Different histologic findings on muscle biopsy have helped in classifying and diagnosing different types of congenital myopathies [1]. Diagnostic accuracy of different rare and congenital diseases has been improved by the advancement in the next-generation sequencing [2]. This has largely replaced the need for more invasive procedures and expedited diagnosing these diseases. It will help in giving proper treatment and in genetic counseling [2].

Small rod-like inclusions in muscle fibers are distinctive features of nemaline myopathy [3]. It has broad clinical spectrum, ranging from mild to severe phenotype [4]. Different genetic mutations can cause nemaline myopathy [5]. Mutations in NEB gene encoding nebulin are considered the most encountered [6]. Nemaline myopathy is underreported in Saudi Arabia. Here, we report a case of nemaline myopathy in a 16-year-old girl using next-generation sequencing. 


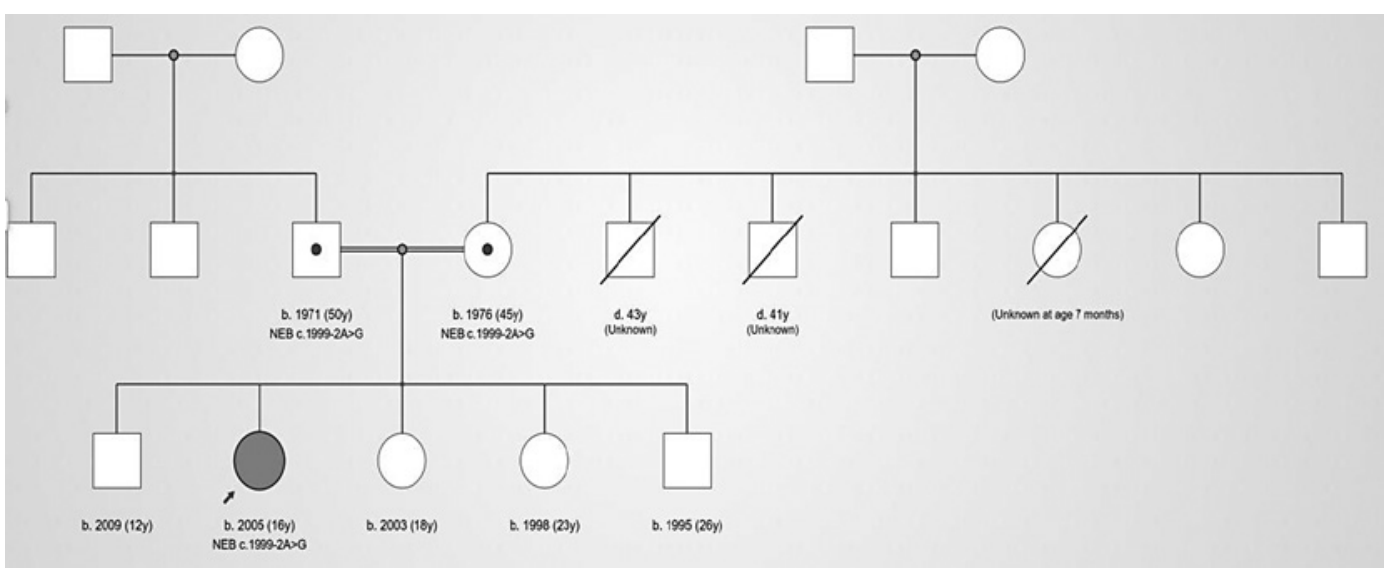

Fig. 1. Family pedigree.

\section{Case Report}

A 16-year-old girl presented in the clinic with her parents complaining of a longstanding history of weakness. She reported that she never had the ability to run. She had difficulty in climbing stairs and in reaching out for things. She denied any history of muscle pain and stiffness. There was no history of diplopia, shortness of breath, or change in urine color. The weakness was not fluctuating and not associated with dysphagia or facial weakness. When asked about dexterity weakness, she said that it is quite difficult when trying to turn the doorknob or opening jars with also infrequent history of dropping things off the hands. She would get some help when standing from seating position. This patient denied any history of using walking aids. There was no history of numbness, burning, or tingling in both upper and lower extremities. As her family was concerned, her parents sought medical advice from the age she was 2 years old. At the age of 14 years, she had second opinion evaluation after which she was labeled as congenital myasthenia. She was started on prednisone and acetylcholinesterase inhibitor. She continued this regimen for more than a year without noticeable benefit. Her parents are consanguineous. She had delayed motor milestones. She sat at 8 months, was able to stand at 15 months, and walked at almost 2 years of age. She never was able to run. She has 2 sisters and 2 brothers with none of them had similar symptoms (Fig. 1). The parents denied similar symptoms in their families. She is a high school student with normal IQ.

On examination, she had a symmetrical face with no ptosis, ophthalmoplegia, or dysmorphic characteristics that could be identified. No muscle wasting or fasciculations in the tongue or extremities were observed. Tone was reduced throughout. Muscle strength assessment showed proximal more than distal weakness more so in the shoulders' abductors and hips flexors $4 / 5$. Fingers abductors were at $4 / 5$ bilaterally. Otherwise, the neurological examination was normal.

Routine blood work, CPK, and vitamin D levels were normal. Thyroid and vasculitis screening were unremarkable. Imaging of the spinal cord was unremarkable. Echocardiography and pulmonary function tests were normal. Nerve conduction evaluation for right upper and lower extremities was normal, but electromyography was myopathic (Tables 1 and 2).

Whole-exome sequencing for the patient and her parents revealed a pathogenic homozygous splice acceptor variant in NEB gene c.1999-2A>G. Both parents were carriers of the same variant. 
Table 1. Electrophysiological data (right arm and leg)

\begin{tabular}{|c|c|c|c|c|c|}
\hline \multicolumn{6}{|c|}{ Nerve conduction studies } \\
\hline nerve & DML, ms & CMAP Amp, mV & DSL, ms & SNAP Amp, $\mu \mathrm{V}$ & Motor CV, m/s \\
\hline Median & $3.9(\leq 4.2)$ & $7(\geq 5)$ & $2.1(\leq 2.4)$ & $17(\geq 15)$ & $49(\geq 48)$ \\
\hline Ulnar & $3.5(\leq 3.6)$ & $6.6(\geq 6)$ & $2.3(\leq 2.4)$ & $18(\geq 15)$ & $52(\geq 48)$ \\
\hline Peroneal & $3.1(\leq 5.8)$ & $5.1(\geq 3)$ & $3.4(\leq 3.5)$ & $6.4(\geq 5)$ & $44(\geq 39)$ \\
\hline Tibial/sural & $4.2(\leq 5.8)$ & $4.7(\geq 4)$ & $3(\leq 3.5)$ & $8(\geq 5)$ & $42(\geq 39)$ \\
\hline
\end{tabular}

Normal values in parenthesis.CMAP, compound muscle action potential; DML, distal motor latency; SNAP, sensory nerve action potential.

Table 2. Needle electromyography

\begin{tabular}{lllllll}
\hline Muscle & INSER & FIB & REC & AMP & DUR & POLY \\
\hline Deltoid & $N$ & 0 & Early & Small & Short & Inc \\
Biceps & $N$ & 0 & Early & Small & Short & Inc \\
First dorsal interosseus & $N$ & 0 & $N$ & $N$ & $N$ & $N$ \\
Iliopsoas & $N$ & 0 & Early & Small & Short & Inc \\
Vastus medialis & $N$ & 0 & Early & $N$ & $N$ & Borderline \\
Tibialis anterior & $N$ & 0 & $N$ & $N$ & $N$ & $N$
\end{tabular}

Insertional activity: $N$, sust, unsust, FIB: $0,1+, 2+, 3+, 4+$, recruitment: $N$, early, reduced, amplitude: $N$, small, big, duration: $N$, short, long, polyphasia: $N$, inc, dec.

She was diagnosed with autosomal recessive nemaline myopathy. The patient was advised to do strengthening and range of motion exercises for the proximal muscles with maintaining normal vitamin D level. She will be followed up in clinic regularly.

\section{Discussion}

Systematic approach to patients presenting with neuromuscular symptoms is warranted. This patient was diagnosed with congenital myasthenia based only on normal CK level and history of weakness. Fluctuating weakness of the skeletal and ocular muscles and asymmetric ptosis are keys in diagnosing myasthenia gravis [7]. None of these symptoms were present in this patient. She was put for more than a year on unnecessary treatments with major side effect profile. Our patient presented with slowly progressive weakness for almost 14 years and has history consanguinity which put congenital muscular dystrophy and myopathy top on the list. Normal CK level is unlikely present in congenital muscular dystrophy in which sarcoplasmic membrane is disrupted [5]. Nerve conduction study was normal in this patient. Electromyography showed myopathic pattern without evidence of muscle fiber irritability, that is, fibrillation potentials and positive sharp waves which make juvenile inflammatory myopathy unlikely. Next-generation sequencing is cost-effective especially when considering sequencing large genes such as $N E B$ gene [3]. Proper history taking and examination are keys in ordering appropriate investigations. Nemaline myopathy is a type of congenital myopathies characterized by varying degrees of generalized body weakness and hypotonia with majority of cases present at birth or early childhood, but sporadic late-onset nemaline myopathy cases have been reported in adults, which can be associated with HIV $[8,9]$. Majority of disease causing are recessive 
variants of $N E B$ gene [10]. This patient was found to have a pathogenic homozygous splice acceptor variant in $N E B$ gene c.1999-2A $>$ G. Splice site mutations are the commonest variants affecting $N E B$ followed by frameshift mutations [10]. As Nemaline myopathy is a heterogenous disease with more severe forms can cause dilated cardiomyopathy and respiratory failure, echocardiography and pulmonary function tests were normal in our patient. We explained to her and her parents' different symptoms of heart failure and respiratory compromise and to come to ER when they feel so. No specific treatment exists for nemaline myopathy rather addressing symptomatic issues [6]. Clinicians should be aware of different phenotypes of neuromuscular disorders and refer atypical cases to more specialized centers.

\section{Acknowledgements}

The author would like to thank the patient and her parents for their cooperation.

\section{Statement of Ethics}

Written informed consent was obtained from the parents of the patient and from the patient for publication of this case report and any accompanying images. The paper is exempt from Ethical Committee approval because it does not meet the DHHS definition of "research."

\section{Conflict of Interest Statement}

The author declares that there is no conflict of interest.

\section{Funding Sources}

The author received no financial support for the case report, authorship, and/or publication of this article.

\section{Author Contributions}

The author collected the data of the case, performed the literature search, wrote the initial manuscript drafts, and completed the revision of the case.

\section{Availability of Data and Material}

All data generated or analyzed during this study are included in this article and its see online suppl. Material; for all online suppl. material, see www.karger.com/doi/10.1159/ 000517898 files. Further enquiries can be directed to the corresponding author. 


\section{References}

1 Ravenscroft G, Bryson-Richardson RJ, Nowak KJ, Laing NG. Recent advances in understanding congenital myopathies. F1000Res. 2018;7:1921.

2 Boycott KM, Vanstone MR, Bulman DE, MacKenzie AE. Rare-disease genetics in the era of next-generation sequencing: discovery to translation. Nat Rev Genet. 2013;14(10):681-91.

3 Cassandrini D, Trovato R, Rubegni A, Lenzi S, Fiorillo C, Baldacci J, et al. Congenital myopathies: clinical phenotypes and new diagnostic tools. Ital J Pediatr. 2017 Nov 15;43(1):101.

4 Laing NG, Dye DE, Wallgren-Pettersson C, Richard G, Monnier N, Lillis S, et al. Mutations and polymorphisms of the skeletal muscle $\alpha$-actin gene (ACTA1). Hum Mutat. 2009;30(9):1267-77.

5 North KN, Wang CH, Clarke N, Jungbluth H, Vainzof M, Dowling JJ, et al. Approach to the diagnosis of congenital myopathies. Neuromuscul Disord. 2014;24(2):97-116.

6 Butterfield RJ. Congenital muscular dystrophy and congenital myopathy. Continuum. 2019;25(6):1640-61.

7 Juel VC, Massey JM. Myasthenia gravis. Orphanet J Rare Dis. 2007;2(1):44.

8 Schnitzler LJ, Schreckenbach T, Nadaj-Pakleza A, Stenzel W, Rushing EJ, Van Damme P, et al. Sporadic lateonset nemaline myopathy: clinico-pathological characteristics and review of 76 cases. Orphanet J Rare Dis. 2017 May 11;12:86.

9 Neuhaus SB, Wallgren-Pettersson C, Bönnemann CG, Schara U, Servais L; Nemaline Working Group. 250th ENMC international workshop: Clinical trial readiness in nemaline myopathy 6-8 september 2019, hoofdorp, the netherlands. Neuromuscular disorders. 2020;30(10):866-75.

10 Lehtokari VL, Kiiski K, Sandaradura SA, Laporte J, Repo P, Frey JA, et al. Mutation update: the spectra of nebulin variants and associated myopathies. Hum Mutat. 2014;35(12):1418-26. 